\title{
Intestinal Neuroendocrine Tumor
}

National Cancer Institute

\section{Source}

National Cancer Institute. Intestinal Neuroendocrine Tumor. NCI Thesaurus. Code C96062.

A well differentiated, low or intermediate grade tumor with neuroendocrine differentiation that arises from the small or large intestine. 